\title{
A New Theory on Electron Wave-Particle Duality
}

\author{
Nader Butto \\ Dgania, Petah Tikva, Israel \\ Email: nader.butto@gmail.com
}

How to cite this paper: Butto, N. (2020) A New Theory on Electron Wave-Particle Duality. Journal of High Energy Physics, Gravitation and Cosmology, 6, 567-578. https://doi.org/10.4236/jhepgc.2020.64038

Received: July 3, 2020

Accepted: August 10, 2020

Published: August 13, 2020

Copyright $\odot 2020$ by author(s) and Scientific Research Publishing Inc. This work is licensed under the Creative Commons Attribution International License (CC BY 4.0).

http://creativecommons.org/licenses/by/4.0/

\begin{abstract}
A theory employing the vortex shape of the electron was presented to resolve the enigma of the wave-particle duality. Conventions such as "particle" and "wave" were used to describe the behavior of quantum objects such as electrons. A superfluid vacuum formed the base to describe the basic vortex structure and properties of the electron, whereas various formulations derived from hydrodynamic laws described the electron vortex circumference, radius, angular velocity and angular frequency, angular momentum (spin) and magnetic momentum. A vortex electron fully explained the associations between momentum and wave, and hydrodynamic laws were essential in deriving the energy and angular frequency of the electron. In general, an electron traveling in space possesses internal and external motions. To derive the angular frequency of its internal motion, the Compton wavelength was used to represent the length of one cycle of the internal motion that is equal to the circumference of the electron vortex. The angular frequency of the electron vortex was calculated to obtain the same value according to Planck's theory. A traveling vortex electron has internal and external motions that create a three-dimensional helix trajectory. The magnitude of the instantaneous velocity of the electron is the resultant of its internal and external velocities, being equal to the internal velocity reduced by the Lorentz factor (whose essence is presented in a detailed formulation). The wavelength of the helix trajectory represents the distance traveled by a particle along its axis during one period of revolution around the axis, resulting in the same de Broglie wavelength that corresponds to the helix pitch of the helix. Mathematical formulations were presented to demonstrate the relation between the energy of the vortex and its angular frequency and de Broglie's wavelength; furthermore, Compton's and de Broglie's wavelengths were also differentiated.
\end{abstract}

\section{Keywords}

Superfluid Vortex, Compton Wavelength, Electron Momentum, Broglie Wavelength, Lorentz Factor, Angular Frequency 


\section{Introduction}

Wave-particle duality is one of the fundamental principles of quantum mechanics which is directly linked to many of its mysteries. According to this theory, light and matter exhibit properties of both waves and particles. The wave characteristic of an electron implicates many of its particle behavior; however, the two-sided nature of electron duality does not allow for it to be observable as a particle and as a wave. Therefore, this duality addresses the inadequacy of conventional concepts, such as "particle" and "wave," to meaningfully describe the behavior of quantum objects.

Light behaves as a wave as it travels through space. However, like a tiny particle, it gives up its energy the moment it reaches its destination. Such a duality behavior is not confined to light; in fact, numerous experiments have supported its existence in photons [1], electrons [2] [3] [4] [5], neutrons [6], atoms and dimers [7], small van der Waals clusters [8] and more recently, C60 fullerenes [9] [10].

The idea of duality is based on a debate on the nature of light and matter dating back to the 17th century, when Christiaan Huygens and Isaac Newton proposed competing theories of light. However, the dilemma between the wave and particle aspects of the various components of matter and radiation arose after Planck's discovery of the quantum of action.

Planck's quantum hypothesis states that a quantum of energy is related to the frequency by the equation $E=h v$, at the same time, the energy is emitted in little packets of energy called quanta, instead of a continuous emission. However, in quantum physics, the wave is not defined-it is a sort of "clouds" of electron orbitals around an atom which are not physical things but representations of probabilities.

In 1923, Louis de Broglie proposed a hypothesis stating that electrons and other discrete bits of matter, then conceived only as material particles, hold properties of waves. Within a few years, de Broglie's hypothesis was tested by a double-slit experiment which demonstrated that the electron stream acts like a light, proving de Broglie correct. In 1928, Neils Bohr announced an understanding of the complementary relation between the wave aspects and the particle aspects of the same phenomenon in what is known as the complementarity principle, reflecting his argument of the non-necessity to use the words "wave" and "particle" at the same time and the prohibition of questions such as "What is light?" and "What is an electron?" According to Bohr, we must confine ourselves into asking how things are observed to behave under a specified set of circumstances, a solution that denies the possibility of saying anything meaningful about a world that is not being observed, and at the same time, a limitation that was completely unacceptable to many physicists, including Einstein.

On the basis of experimental evidence, Einstein made an independent proposal of a sort of revival of the corpuscular theory of light, including the concept of the quantum of action in the form of energy or light quanta. In 1905 he first 
showed that light, then considered a form of electromagnetic waves, must also be thought of as particle-like, localized in packets of discrete energy. In his theory of photoelectric effect, he posited that when light is shown on certain objects, electrons will be released; if a photon of an energy greater than that of the electron hits a solid, that electron will be emitted. Einstein's theory of photoelectric effect contributed largely to de Broglie's theory and was a proof that waves and particles could overlap. Moreover, Compton's observations of his Compton effect (1922) could be explained only if light has a wave-particle duality. Later, in 1927, the wave nature of electrons was experimentally established by Clinton Davisson and Lester Germer and independently by George Paget Thomson [11].

The problem of wave-particle duality remains unsolved until this day. In quantum mechanics, an electron may appear to us as a particle or as a wave depending on how we "look" at it, and mainly because it is considered as a cloud of structure-less point. There is no theory at present that describes the shape or predicts the size of an electron, its mass, or its charge, or that quantifies the particle in a meaningful calculation or the relationship between the electron as a particle and electron as a wave.

To provide a suitable interpretation and resolve this ostensible paradox, a new theory is presented to demonstrate that the wave-particle duality can be explained by considering the wave as a trajectory of a rotating electron that travels in space. Here, the vacuum is considered a superfluid and the electron an irrotational vortex made up of this fluid. Applying the hydrodynamic laws, a formulation to measure the circumference of the vortex gives the same value of Compton wavelength, whereas an equation to measure the angular frequency of the vortex demonstrates that it has the same wave frequency as the electron according to Planck's theory. Thus, the local physical reality of the electron as a vortex determines the results of local measurements.

\section{The Electron Structure}

According to current experiments and theories, the electron is a structure-less, point-like object. It is not made of anything else and its entire mass is concentrated in its extension-less center. Nonetheless, the electron displays properties that normally result from an extended structure, namely, angular momentum (spin), magnetic moment, and some sort of an internal oscillation. In 1928, when Paul Dirac presented the wave function of the electron in the Dirac equation, it became clearer that there must not only be an internal oscillation but also some internal motion at the speed of light.

Another article proposed that the vortex shape of the electron is a condensation of the vacuum which provides the correct relationship between the parameters of the electron-mass, density volume, time, constant angular momentum (spin), electric charge, and magnetic moment. A brief description of the electron structure is shown in Figure 1. 


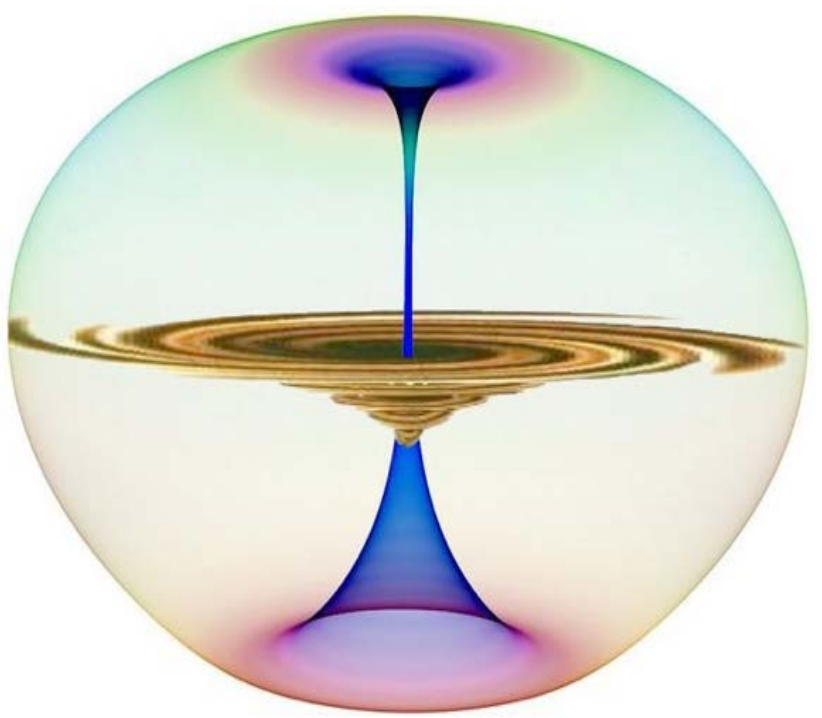

Figure 1. Artistic representation of the magnetic field around the electron vortex.

In the electron vortex, virtual photons flow in a spiraling downward motion in the funnel due to the pressure gradient normal to the vortex center and that acts along the central axis of the vortex spin. It follows down moving up around the vortex and returns from the upper side of the central axis in the mouth of the vortex generating the magnetic momentum. The vertical magnetic pressure gradient is normal to the horizontal electrostatic pressure gradient created by the vortex and acts along the central axis of the spin. The three-dimensional magnetic field has a negative pole that sucks the energy from the vacuum and a positive pole that pulls the energy from the positive magnetic pole. Furthermore, the rotating vortex exercises a rotation effect on the magnetic field, resulting in two movements $90^{\circ}$ on from the other.

The acting electrostatic and magnetic forces become interlinked in self-balancing feedback loops that provide great stability to the vortex structure shape of the particle as a whole. These are the two forces that generate movement and create currents. The currents are the avenues of movement made up of Higgs particles and surrounding these are spaces or crevices through which currents of lesser density comprising Higgs particles flow.

Hydrodynamic laws are applied to describe the behavior of a single electron in space, and to describe its dynamics as a vortex particle in connection with de Broglie's particle-wave hypothesis and Planck's theory, its energy and wave frequency, vortex angular frequency and finally, the electromagnetic wave characteristics and free electron travel in space.

\section{The Vortex Model and De Broglie's Hypothesis}

The essence and physical relationship between a particle and a wave remains an unresolved problem in physics. Louis de Broglie developed a hypothesis [12] relating the dual wave and particle behavior that can be applied to electrons. 
In his hypothesis, he first used Einstein's equation of matter and energy

$$
E=m c^{2},
$$

where $E$ represents the energy of matter, $m$ its mass, and $c$ the speed of light.

He then proceeded with Planck's theory, which states that every quantum of a wave has a discrete amount of energy given by Planck's equation

$$
E=h f \text {, }
$$

where $E$ represents energy, $h$ Plank's constant $\left(6.62607 \times 10^{-34} \mathrm{~J} \cdot \mathrm{s}\right)$ and $f$ frequency.

According to him, the energies produced in both equations should be the same; thus,

$$
m c^{2}=h f,
$$

Substituting $c / \lambda$ for $f$, de Broglie arrived at a final expression relating the wavelength $\lambda$ and particle momentum $m c$ with speed of light:

$$
m c^{2}=h c / \lambda,
$$

where two relationships can be derived:

$$
\lambda=h / m c
$$

and

$$
h=m c \lambda .
$$

In this de Broglie wavelength formula relating $\lambda$ and $m c$ of a wave/particle [13], supposed that the mass of the electron is $m_{e}=9.109 \times 10^{-31} \mathrm{~kg}, h=6.63 \times$ $10^{-34}$ and $c=2.998 \times 10^{8}$, then the derived wavelength will be $2.42779 \times 10^{-12} \mathrm{~m}$, which corresponds to the Compton wavelength.

However, this relation does not explain the mechanism that connects the wave with the particle. By contrast, the electron as a vortex explains fully the association between momentum and wave. The rate of rotation of the fluid in the irrotational vortex is greatest at the center and decreases progressively with distance from the center until no gradient pressure remains on the boundaries of the vortex where the flow is laminar and the friction null. However, the speed of rotation in every point of the vortex is the speed of light $c$.

In hydrodynamics, the velocity of the fluid element instantaneously passing through a given point in space in a vortex with radius $r$ is constant in time, thus, the circulation or the vorticity $\Gamma$ is defined by

$$
\Gamma=2 \pi r c,
$$

Because $\Gamma m$ is a conserved momentum, $2 \pi r c m$ is constant, which corresponds to the Planck constant.

Accordingly,

$$
\begin{gathered}
\Gamma_{e}=2 \pi r_{e} c, \\
h=\Gamma_{e} m_{e}
\end{gathered}
$$

and

$$
\Gamma_{e}=h / m_{e}=\text { constant } .
$$


Supposed the electron mass is $9.109 \times 10^{-28} \mathrm{~g}$ and Planck's constant is $6.6262 \times$ $10^{-27} \mathrm{erg} \cdot \mathrm{s}$, then $h / m_{e}=7.274$,

From these values, the radius of the electron can be calculated as

$$
r_{e}=\frac{\Gamma_{e}}{2 \pi c}=3.863509856 \times 10^{-13} \mathrm{~m}^{20} .
$$

If

$$
2 \pi r c m=h=m c \lambda,
$$

then

$$
2 \pi r=\lambda
$$

Knowing that the radius of the vortex is the Compton radius, $3.86 \times 10^{-13} \mathrm{~m}$, the circumference is $2 \pi r=2.42408 \times 10^{-12} \mathrm{~m}$, which agrees well with the CODATA 2014 [14] value for the Compton wavelength of the electron $2.4263102367(11) \times 10^{-12} \mathrm{~m}$ [15]. Therefore, we can conclude that the Compton wavelength is produced by one rotation cycle of the electron vortex.

\section{Vortex Angular Frequency and Electron Frequency}

According to Planck's theory, photons of frequency $f$ produces an energy

$$
E=h f_{e} .
$$

The frequency of the electron is equivalent to the frequency of the photon having the same energy thus,

$$
f_{e}=E / h,
$$

where $f_{e}=E / h=0.511 \mathrm{MeV} / h=8.1866 \times 10^{-7} \mathrm{ergs} / \mathrm{h}$. Therefore, $f_{e}=1.2355 \times$ $10^{20}$ cycles/s.

Another way to describe the vortex is by its angular velocity. Being material particles, vortices share similar mechanical properties with waves. All streamlines of the vortex tubes rotate around their axis and the vortices have a measurable rotating angular frequency that can be described in units of time (rotations per second).

Because the rotational velocity $\omega$ of the vortex is

$$
\omega=\frac{c}{r}=2 \pi f,
$$

the frequency $f$ can be expressed as

$$
f=\frac{c}{2 \pi r} .
$$

Assuming $V=c=2.889 \times 10^{8}$ and $2 \pi r=2.42408 \times 10^{-12} \mathrm{~m}$, the frequency is $2.998 \times 10^{8} / 2.42408 \times 10^{-12}=1.2367 \times 10^{20}$ cycles $/ \mathrm{s}$, which coincides with the electron frequency derived from Planck's equation.

\section{Relation between the Energy of the Vortex and Its Frequency}

In hydrodynamics, the force $F$ that moves the vortex is directly related to the 
pressure that creates the vortex (or the so-called dynamic pressure $P_{d}$ ) and the area $A$ :

$$
F=P_{d} A
$$

The $P_{d}$ representing the fluid kinetic energy is given by

$$
P_{d}=\frac{1}{2} \rho v^{2},
$$

where $\rho$ and $v=c$ represents the density and velocity of the fluid.

Therefore, the internal force of the vortex can be expressed by

$$
F=\frac{1}{2} \rho c^{2} A .
$$

The area of the vortex is approximately a circle, and its radius when stretched will cause the vortex radius to double in size; therefore, $A=2 \pi r^{2}$. Correspondingly,

$$
F=\rho c^{2} \pi r^{2} .
$$

If the right-hand side of the equation is multiplied and divided by a time $t$, then

$$
F=\frac{\rho c t \pi r^{2} c}{t} .
$$

The quantity ( $c t$ ) is equivalent to the distance $L, L \pi r^{2}$ is equivalent to the volume $Q, \rho Q$ is equivalent to the mass $m$, and $1 / t$ is equivalent to the frequency $f$. Therefore,

$$
F=m c f .
$$

The energy of the rotating electron around its axis is $E=$ force $\times$ distance. As the electron rotates a distance of $2 \pi r$ in one cycle,

$$
E=2 \pi r c m f,
$$

which gives the rest energy of the vortex electron. Furthermore, the frequency can be derived as

$$
f=\frac{E}{2 \pi r c m} .
$$

Since

$$
2 \pi r c m=h,
$$

then

$$
f=\frac{E}{h} .
$$

In this case, the frequency indicates the number of passages of a single electromagnetic wave within one second of time. Planck's constant is the energy found within one cycle.

\section{Flow of Free Electron in Space}

De Broglie's hypothesis also states that each portion of energy with a rest mass 
$m_{0}$ may associate a periodic phenomenon of the frequency $f_{0}$, such that $h f f_{0}=$ $m_{0} c^{2}$, where $f_{0}$ is the rest mass frequency [15].

He used the special theory of relativity to find that, in the frame of the observer of the electron energy packet moving with a velocity $V$, its frequency was apparently reduced to

$$
f=f_{0}\left(1-\frac{v^{2}}{c^{2}}\right)^{\frac{1}{2}}
$$

where $\left(1-\frac{v^{2}}{c^{2}}\right)^{\frac{1}{2}}$ is the Lorentz factor, a factor by which time, length and relativistic mass change for an object while it moves. The expression appears in several equations of special relativity and arises in derivations of the Lorentz transformations. However, the mechanism from which this factor was derived is not known.

However, if

$$
m c^{2}=h f_{0}\left(1-v^{2} / c^{2}\right)^{1 / 2}, \quad f_{0}=c / \lambda \text { and } m c^{2}=(h c / \lambda)\left(1-v^{2} / c^{2}\right)^{1 / 2},
$$

then

$$
\lambda=(h / m c)\left(1-v^{2} / c^{2}\right)^{1 / 2}=h / p,
$$

also known as the "de Broglie wavelength" for a particle moving at $v$ to a fixed observer [16].

However, the mechanism that associates the mass with frequency is not explained and the origin of Lorentz factor is not clear.

The electron as a vortex in free space will never be still; it will move continuously around its axis. In order to complete one cycle, the rotating electron vortex needs to complete a distance of $2 \pi r$ which is equivalent to $c t$, with $t$ being the time needed to complete one cycle and $c$ the speed of light.

Consequently,

$$
2 \pi r=c t
$$

if

$$
\frac{1}{t}=f
$$

The frequency of the rest electron is

$$
f_{0}=\frac{c}{2 \pi r}
$$

with the electron vortex circumference $2 \pi r$ corresponding to the Compton wavelength $\lambda$.

Moreover, because

$$
\begin{gathered}
2 \pi r c m=h=m c \lambda, \\
\lambda=\frac{h}{m c} .
\end{gathered}
$$


Every microparticle is endowed with two types of motion, an internal motion and an external motion. A vortex electron travelling in space has internal and external velocities. The internal velocity $V_{\perp}$ is in a plane normal to the external parallel velocity $V_{/}$. The magnitude of the instantaneous velocity $v_{0}$ of the electron is the resultant of its internal and external velocities, which is always equal to " $c$," the velocity of light in free space.

As shown in Figure 2, due to Magnus effect, the vortex deviates an angle a and a vortex drift on a three-dimensional helix trajectory.

The helix can be considered as a three-dimensional wave whose pitch is equivalent to the "wavelength". The pitch of the helix is the distance travelled by the particle around the axis in one period of revolution.

The angular velocity is a vector whose magnitude measures the rate at which the radius sweeps out an angle and whose direction shows the principal axis of rotation.

Thus, the velocity $v_{0}$ of the electron of mass $m$ with respect to the origin $\mathrm{O}$ is separated with trigonometric functions and can be resolved into components parallel to $\left(V_{/ I}\right)$ and perpendicular to $\left(V_{\perp}\right)$ the radius vector $r$. Here, $V_{/ /}$is the longitudinal velocity while $V_{\perp}$ is the rotational velocity of the electron around its axis as presented in Figure 3.

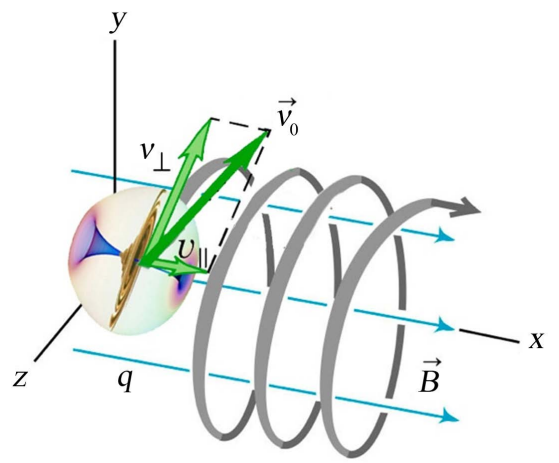

Figure 2. Three-dimensional helix electron trajectory connecting the particle with the wave.

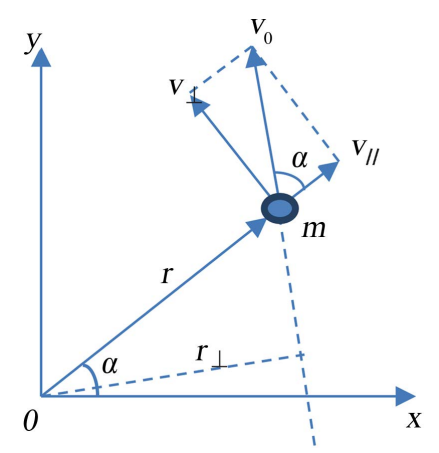

Figure 3. In three-dimensional space, the vector $\boldsymbol{r}$ of a moving particle is the radius vector from the origin. The angular velocity is a vector whose magnitude measures the rate at which the radius sweeps out an angle and whose direction shows the principal axis of rotation and is given by the right-hand rule. 
The internal rotation velocity $V_{\perp}$ of the vortex is determined as

$$
v_{\perp}=\left(v_{0}^{2}-v_{/ /}^{2}\right)^{1 / 2} \text {. }
$$

The angle $\alpha$ can be determined by the trigonometric function

$$
\sin \alpha=v_{\perp} / v_{0}=\left(v_{0}^{2}-v_{/ /}^{2}\right)^{1 / 2} / v_{0}=\left(\left(v_{0}^{2}-v_{/ /}^{2}\right) / v_{0}^{2}\right)^{1 / 2}=\left(1-v_{/ /}^{2} / v_{0}^{2}\right)^{1 / 2} .
$$

Assuming $v_{0}=c$ and $V_{/ /}=v$,

$$
\sin \alpha=\left(1-v^{2} / c^{2}\right)^{1 / 2},
$$

which is the origin of the Lorentz factor.

Although the electron's resultant velocity with respect to an observer is given by $c$, its internal and external velocities would be different according to this observer since there is an additional velocity $v$ between the frames.

The changes in the internal velocity $c$ due to the external motion of the electron is very small for low external velocities. However, external velocities very close to " $c$ " bring about significant changes in the internal velocities. For example, the electron vortex "at rest" with respect to an observer $S$ has an internal velocity " $c$," but the same electron when observed from a frame $S^{\prime}$ moving with a relative velocity $v$ with respect to $S$ will have an internal velocity $c /\left(1-v^{2} / c^{2}\right)^{1 / 2}$ which is less than " $c$ ".

Moreover, the angular momentum can be considered as a rotational analogy of the linear momentum $p$ which is directly proportional to mass $m$ and linear speed $v_{0}$ :

$$
p=m v_{0} .
$$

The electron's angular momentum depends on its instantaneous velocity $v_{0}$ which in turn depends on the internal motion reduced by Lorentz factor as described in

$$
v_{0}=v_{\perp} / \sin \alpha=c / \sin \alpha=c /\left(1-v^{2} / c^{2}\right)^{1 / 2} .
$$

From the relation $\lambda=h / p=\left(h / m v_{0}\right)$ with the angular momentum, the wavelength $\lambda$ of the travelling electron can be expressed in terms of

$$
\lambda=\left(h / m v_{0}\right)=(h / m c) /\left(1-v^{2} / c^{2}\right)^{1 / 2} .
$$

Considering the mass of the electron travelling at $1 \times 10^{5}$ meters per second, $\lambda$ $\sim 7.3 \times 10^{-9} \mathrm{~m}$, or approximately the radial size of an atom. This proves that the de Broglie wavelength is completely different from the Compton wavelength, as illustrated in Figure 4.

\section{Conclusions}

The electron wave-particle properties can be accurately described using classical laws of the Newtonian mechanics that can exhibit particle and wave properties simultaneously. The electron is treated as a superfluid irrotational vortex, thus, its local physical reality as a vortex determines the results of local measurements. 


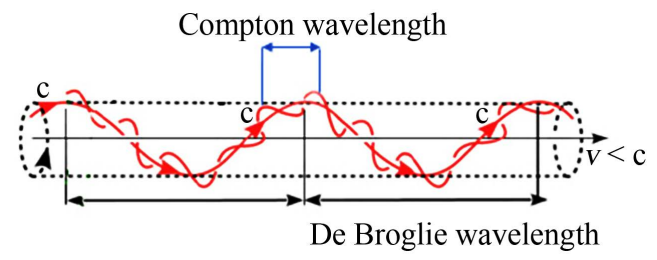

Figure 4. Compton wavelength is generated by one cycle or rotation around the electron vortex axis while de Broglie wavelength is the pitch of the helix generated by an electron travelling in space.

Hydrodynamic laws are applied to study the behavior of such a vortex which has internal rotation, angular momentum, angular speed, and angular frequency. The electron is endowed an internal motion and an external motion, the former being a circular motion in a plane with a radius characteristic of the mass of the electron and produces a Compton wavelength equal to the circumference of the electron vortex. With the combination of the internal and external motions caused by the Magnus effect, the electron drifts on a three-dimensional wave attaining a pitch equivalent to the de Broglie wavelength. The internal rotational motion of the electron travelling in space at a relatively low speed attains the speed of light speed, but is reduced by the Lorentz factor with the electron travelling at high speeds. The magnitude of the instantaneous velocity of the particle is the resultant of its internal and external velocities, and is equal to the internal velocity reduced by the Lorentz factor.

Therefore, the de Broglie wavelength represents the wavelength of a threedimensional helix derived from the electron momentum equation whereas the Compton wavelength represents the rotation of one cycle of the internal motion derived from the circumference vortex equation. In this regard, the electron frequency derived from Planck's equation represents the angular frequency of the rotating electron vortex and has a fixed value while de Broglie's wavelength represents the helix wave frequency which is variable and dependent on the electron's external velocity.

By postulating an electron vortex structure with internal rotation motion, we developed a simple "physical" theory combining elegantly the mechanics with the mathematics that might improve our understanding of the mysteries of nature, i.e., "super conductivity," "quantum mechanical tunneling," and that can explain double-slit experiments. Future experimental studies are needed to confirm the vortex structure of the electron. The electron structure as well as the origin of its mass, energy, and electric charge will be discussed separately in different papers.

\section{Acknowledgements}

The author would like to thank Enago (http://www.enago.com) for the English language review.

This research did not receive any specific grant from funding agencies in the public, commercial, or not-for-profit sectors. 


\section{Conflicts of Interest}

The author declares no conflicts of interest regarding the publication of this paper.

\section{References}

[1] Taylor, G.I. (1909) Interference Fringes with Feeble Light. Proceedings of the Cambridge Philosophical Society, 15, 114-115.

[2] Davisson, C.J. and Germer, L.H. (1927) The Scattering of Electrons by a Single Crystal of Nickel. Nature, 119, 558-560. https://doi.org/10.1038/119558a0

[3] Merkli, P.G., Missiroli, G.F. and Pozzi, G. (1976) On the Statistical Aspect of Electron Interference Phenomena. American Journal of Physics, 44, 306. https://doi.org/10.1119/1.10184

[4] Missiroli, G.F., Pozzi, G. and Valdre, U. (1981) Electron Interferometry and Interference Electron Microscopy. Journal of Physics E, 14, 649. https://doi.org/10.1088/0022-3735/14/6/001

[5] Tonomura, A., Endo, J., Matsuda, T., Kawasaki, T. and Ezawa, H. (1989) Demonstration of Single-Electron Buildup of an Interference Pattern. American Journal of Physics, 57, 117. https://doi.org/10.1119/1.16104

[6] Halban, H. and Preiswerk, P. (1936) Preuve expérimentale de la diffraction des neutrons. Comptes Rendus de 1 Académie des Sciences, 203, 73-75.

[7] Estermann, I. and Stern, O. (1930) Beugung von Molekularstrahlen. Zeitschrift für Physik, 61, 95-125. https://doi.org/10.1007/BF01340293

[8] Schollkopf, W. and Toennies, J.P. (1994) Nondestructive Mass Selection of Small van der Waals Clusters. Science, 266, 1345-1348. https://doi.org/10.1126/science.266.5189.1345

[9] Arndt, M., Nairz, O., Vos-Andreae, J., Keller, C., van der Zouw, G. and Zeilinger, A. (1999) Wave-Particle Duality of C60 Molecules. Nature, 401, 680-682. https://doi.org/10.1038/44348

[10] Nairz, O., Arndt, M. and Zeilinger, A. (2003) Quantum Interference Experiments with Large Molecules. American Journal of Physics, 71, 319-325. https://doi.org/10.1119/1.1531580

[11] Davisson, C.J. and Germer, L.H. (1928) Reflection of Electrons by a Crystal of Nickel. Proceedings of the National Academy of Sciences of the United States of America, 14, 317-322. https://doi.org/10.1073/pnas.14.4.317

[12] Feynman, R. (1990) QED the Strange Theory of Light and Matter. Penguin, London, 84.

[13] de Broglie, L. (1923) Radiation-Waves and Quanta. Comptes Rendus, 177, 507-510, 548. (In French)

[14] CODATA (2014) Value for Compton Wavelength for the Electron from NIST.

[15] de Broglie, L. (1923) Waves and Quanta. Nature, 112, 540. https://doi.org/10.1038/112540a0

[16] de Broglie, L. (1960) Nonlinear Wave Mechanics: A Causal Interpretation. Elsevier, Amsterdam. 